\title{
Microstructural Characterization of Biosynthesized ZnO Nanostructures Using Jatropha dioica Aqueous Extract
}

Villanueva-Ibáñez Maricela ${ }^{1}$, González Montes de Oca Roel ${ }^{2}$, Camargo Pérez Victoria Perla ${ }^{3}$, OlveraVenegas Patricia Nayeli ${ }^{1}$, Armando Irvin Martínez Pérez ${ }^{4}$, Edgar Ernesto Vera Cárdenas ${ }^{5}$, FloresGonzález Marco Antonio ${ }^{1}$

1. Nanotechnology, new materials and systems for health, industry and alternative energies, Universidad Politécnica de Pachuca, Hidalgo, México.

2. Mechatronics Department, Universidad Politécnica de Pachuca, Hidalgo, México.

3. Dirección de investigación, innovación y posgrado, Universidad Politécnica de Pachuca, Hidalgo, Mx.

4. Automotive Mechanics Department, Universidad Politécnica de Pachuca, Hidalgo, México.

5. Dirección de posgrado, Instituto Tecnológico de Pachuca, Hidalgo, México.

Due to its considerable applications in optoelectronic devices, photocatalysis or antimicrobial agent, the manufacture of zinc oxide $(\mathrm{ZnO})$ has been widely studied by several processes, among the most recent are those based on biological systems or their derivatives [1-4], in which the environmental impact is reduced and stimulate the implementation of nanostructured materials in applications compatible with the physiological environment. In biological methods it is possible to use microorganisms, plants or their derivatives to accomplish the reduction of the precursor salts of the material of interest. By using plants, one option is to take advantage of its aqueous extract since it contains antioxidants that work as reducing agents in the formation of $\mathrm{ZnO}$, giving rise to nanostructures with morphology depending on the type and volume of the plant extract, temperature and $\mathrm{pH}[5]$.

In this study, the microstructural characterization of nanostructured $\mathrm{ZnO}$ biosynthesized in aqueous medium with $J$. dioica extract was performed. To obtain it, the aqueous extract of $J$. dioca was put in contact with a zinc acetate solution $0.1 \mathrm{M}$ as the oxide precursor salt, the $\mathrm{pH}$ of the synthesis medium was adjusted to a value of 11 with a $\mathrm{NaOH}$ solution $2 \mathrm{M}$ and deionized water was added until reaching a final volume of $45 \mathrm{~mL}$. The synthesis remained in constant agitation at a temperature of $40^{\circ} \mathrm{C}$ for $4 \mathrm{~h}$. Once the synthesis time had elapsed, the nanostructures were recovered by vacuum filtration, washed with deionized water and then dried at room temperature. The powder obtained was characterized by complementary analysis where UV-vis absorption spectroscopy indicated the characteristic absorption of $\mathrm{ZnO}$ and by infrared spectroscopy with Fourier transform it became evident that the nanostructures are still with organic matter contents coming from the medium of synthesis [6] (Figures 1a and 1b).

By X-ray diffraction, the crystalline structure of the material was determined using a $\mathrm{Cu}-\mathrm{K} \alpha 1$ $(\lambda=0.15406 \mathrm{~nm})$ radiation with a scanning speed of 7 steps/minute and an angle of $5^{\circ}$. Diffractions located in $2 \theta$ at $31.90^{\circ}, 34.54^{\circ}, 36.42^{\circ}, 47.61^{\circ} 56.71^{\circ}, 62.95^{\circ}, 66.39^{\circ}, 67.99^{\circ}$ and $69.18^{\circ}$, assigned to the crystallographic planes (100), (002), (101), (102), (110), (103), (200), (112) and (201) correspond to the hexagonal wurtzite-type structure (Figure 1c). On the other hand, by a scanning electron microscope (SEM) JEOL JSM6010-LA, the morphological characterization of $\mathrm{ZnO}$ was determined under high vacuum with an acceleration of $20 \mathrm{KV}$ and a spot size of the electron beam in 30 . The micrograph in Figure 2 shows semi-spherical particles and triangular faces forming mixed structures with a tendency to join several polyhedral through a central semi-sphere. By means of an X-ray detector with a beryllium window, a dispersed energy spectrum (EDS) was obtained observing the characteristic ZnO peaks and an EDS mapping showed a uniform dispersion of its main elements (Zn, O) (Figures 3a -d). 
For the observation in SEM, the biosynthesized $\mathrm{ZnO}$ was fixed to a portion of graphite conductive tape in a stuff without applying plasma cleaning or gold coating. The obtained results show the direct morphological observation of $\mathrm{ZnO}$ even presenting an important load of organic matter by secondary electrons with the sample inclined at minus $10^{\circ}$, which is attributed to a low interaction between the primary electron beam and the $\mathrm{ZnO}$, besides show enough conductivity to avoid an electrostatic cloud in the observation area.

\section{References:}

[1] N. Jayarambabu, K. Venkateswara Rao, V. Rajendar, Materials Letters 211 (2018), p. 43.

[2] Thenmozhi Karnan, Stanly Arul Samuel Selvakumar, Journal of Molecular Structure 1125 (2016), p. 358.

[3] R. Dobrucka, J. Dugaszewska, Sandi Journal of Biological Sciences, 23 (2016), p. 517.

[4] Deepali Sharma et al, Journal of Photochemistry \& Photobiology, B: Biology 162 (2016), p. 199.

[5] Ahmed, S., Ali, S., \& Ikram, S. (2017). Journal of Photochemistry \& Photobiology, B: Biology 166 (2017), p. 272.

[6] Bhuyan, T. et al, Materials Science in Semiconductor Processing 32 (2015), p. 55.
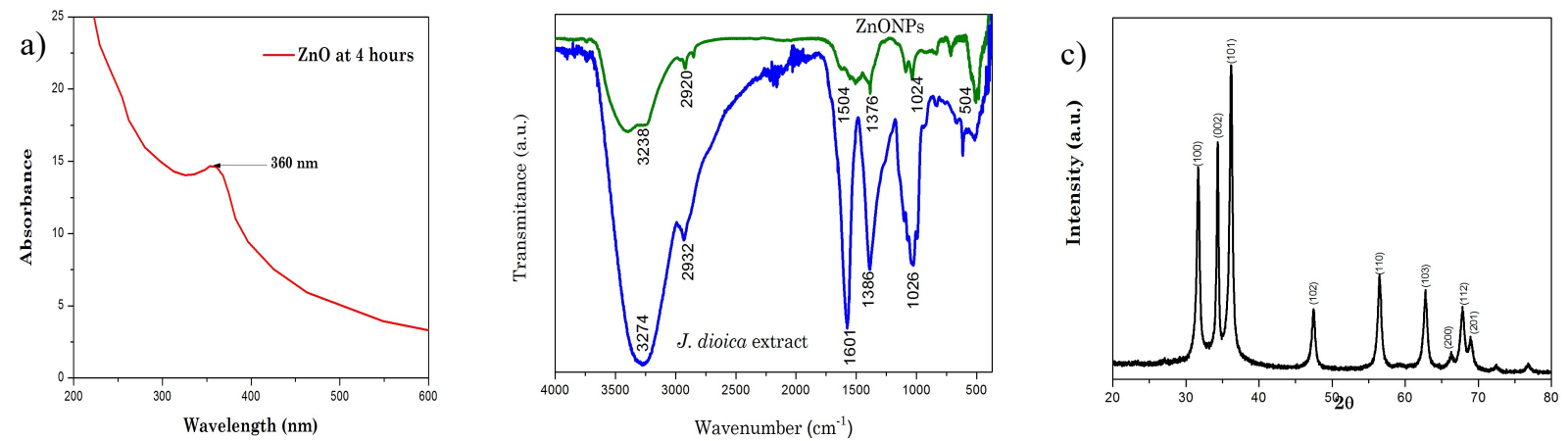

Figure 1. $\mathrm{ZnO}$ biosynthesized with aqueous extract of $J$. dioica a) UV-vis with maximum absorption at $360 \mathrm{~nm}$; b) FT-IR showing the strong load of organic matter coming from the extract in the nanostructures; b) XRD of its wurtzite hexagonal crystal structure.

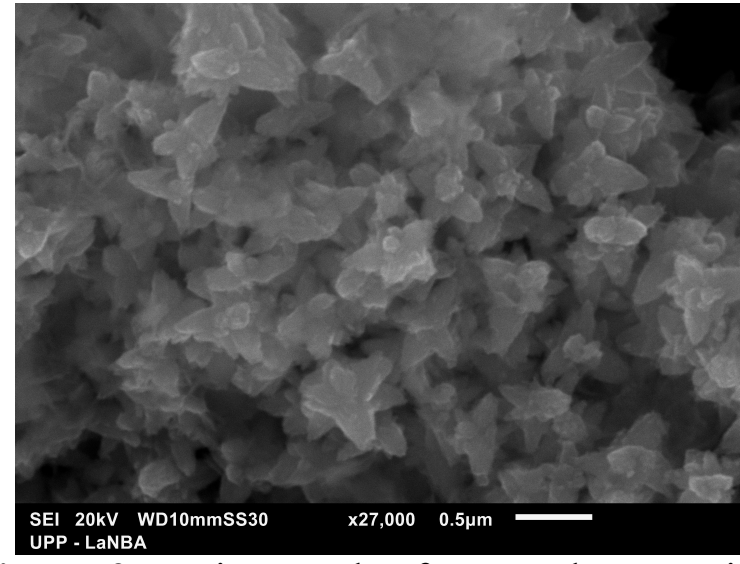

Figure 2. Micrograph of $\mathrm{ZnO}$ where particle profiles between 80 and 500 nanometers are appreciated.
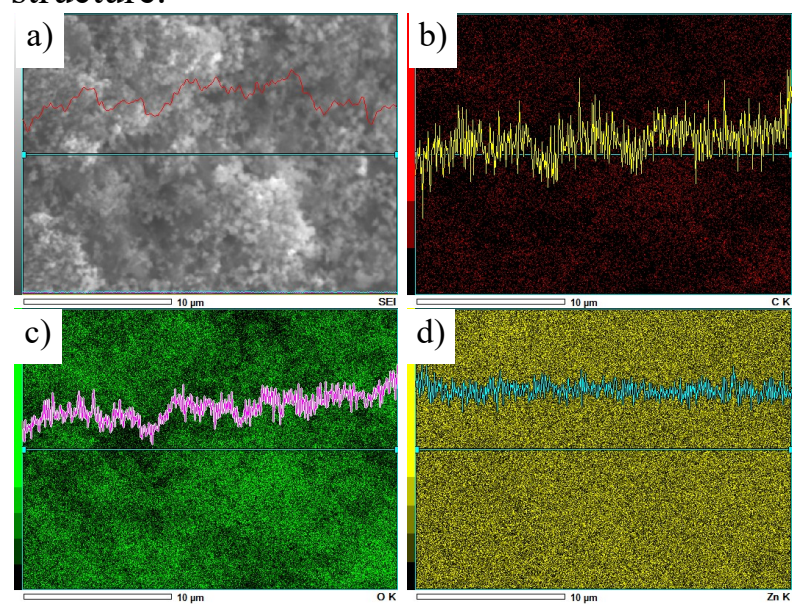

Figure 3. a) Micrograph of the EDS analysis area and EDS map with distribution of (b) Carbon, (c) Oxygen and (d) Zinc. 